\title{
L'apprendistato in Italia: una misura di politica attiva ancora poco valorizzata
}

\author{
Sandra D’Agostino \\ INAPP
}

$\underset{\text { SilvaPP }}{\text { Sia Vaccaro }}$

\begin{abstract}
Da qualche anno l'Italia punta sull'apprendistato quale misura di politica attiva finalizzata a favorire la transizione scuola-lavoro dei giovani. In particolare, l'interesse si è concentrato sulle forme di apprendistato legate ai titoli di studio, ispirate alle migliori esperienze europee. L'analisi dell'evoluzione del sistema duale in Francia, Germania, Austria e Svizzera fornisce importanti indicazioni su come orientare gli interventi; I'analisi dell'esperienza italiana mostra alcune fragilità del disegno di policy e consente di avanzare delle proposte di miglioramento.
\end{abstract}

For some years, Italy has been focusing on apprenticeships as an active policy measure aimed at encouraging youth school-to-work transition. In particular, interest has focused on the forms of apprenticeship linked to general education qualifications, inspired by the best European experiences. The analysis of the transformation of the dual systems in France, Germany, Austria, and Switzerland provides important suggestions on how to orient interventions; the analysis of the Italian experience shows some fragility of the policy design and makes proposals for improvement.

DOI: 10.53223/Sinappsi_2021-02-6

\section{Citazione}

D’Agostino S., Vaccaro S. (2021), L'apprendistato

in Italia: una misura di politica attiva ancora

poco valorizzata, Sinappsi, XI, n.2, pp.120-133
Parole chiave

Transizione scuola-lavoro

Apprendistato

Giovani

\section{Keywords}

School-to-work transition

Apprenticeship

Young people

\section{Introduzione}

Negli ultimi decenni le grandi trasformazioni dei sistemi economici hanno messo in difficoltà $i$ giovani nel percorso di inserimento nel lavoro. La globalizzazione e l'innovazione, come pure le crisi che hanno attraversato le economie tra il 2007 e il 2011, hanno determinato una crescita della disoccupazione giovanile che ha messo in allarme i policymaker. La pandemia da Covid-19 ha innescato una crisi economica che, ancora una volta, colpisce soprattutto i giovani e, in particolare, coloro che si trovano in una condizione di maggiore fragilità.
I Paesi europei hanno cercato di fronteggiare la crescente disoccupazione giovanile adottando misure di politica attiva finalizzate a sostenere l'inserimento o re-inserimento lavorativo; fra queste, alcune guardano al sistema di formazione come ambito che può offrire un prezioso contributo per accrescere l'occupabilità dei giovani e contrastare il mismatch educativo. Anche I'Italia negli ultimi venti anni ha più volte puntato sul rilancio dell'apprendistato, anche se fino ad ora con risultati non particolarmente significativi, e il PNRR si muove nella stessa direzione proponendo misure di potenziamento dello strumento.

Il testo è stato chiuso a marzo 2021. 
Il contributo sintetizza i risultati di un lavoro di ricerca che ha inteso approfondire le ragioni che supportano l'introduzione di un sistema duale come strumento che favorisce l'inserimento dei giovani. Analizzando le esperienze dei Paesi con un sistema duale solido e diffuso (Germania, Svizzera, Austria), insieme a quelle della Francia che, pur non vantando una grande tradizione in questo campo, ha da qualche tempo posto l'apprendistato al centro del proprio impegno riformatore, ci si è interrogati sulle condizioni che possono assicurare o mantenere il successo di tale opzione formativa che segue traiettorie di evoluzione influenzate dai cambiamenti prodotti dai megatrend globali e dalle misure di policy adottate dai Paesi per gestirli.

Esaminando il recente tentativo di costruire una 'via italiana al duale' alla luce delle evidenze rilevabili dai contesti europei, emerge la necessità di inquadrare l'avvio di una nuova proposta formativa in un progetto più ampio di rafforzamento di tutto il sistema di formazione professionale iniziale, che impone un modello di governance multilivello e la ridefinizione del ruolo che i vari stakeholder sono chiamati a ricoprire. Le specificità territoriali del nostro Paese impongono di accompagnare la diffusione di tale dispositivo con strumenti di supporto a intensità variabile, per evitare che le misure di policy finiscano per beneficiare solo i territori già ricchi di opportunità formative e occupazionali, aumentando il divario fra le varie regioni. Come pure è necessario valutare in maniera più attenta i potenziali effetti dell'apprendistato in relazione alle disuguaglianze sociali, alla luce dei dati che evidenziano una minore capacità inclusiva dei gruppi più vulnerabili nei Paesi che vantano i sistemi duali più consolidati.

\section{Giovani e lavoro: il ruolo delle politiche attive per superare le fasi di crisi}

Trascorso un anno dalla proclamazione dello stato di emergenza in Italia per via dell'insorgere della pandemia da Covid-19, alcuni dati consentono di fare un'analisi degli effetti generati sull'economia e sul mercato del lavoro. Secondo le prime stime, la riduzione del PIL nel 2020 è stata pari al 6,3\% nell'UE. In tutti i Paesi europei il PIL ha subito una contrazione; I'Italia mostra una performance peggiore di quella media, con una variazione negativa pari a $-8,8 \%$ (Commissione europea 2021).
L'avvio massiccio della campagna vaccinale consente di formulare previsioni ottimistiche per il futuro con una crescita stimata del 3,7\% per l'area UE nel 2021.

L'impatto della pandemia sul mercato del lavoro è senza precedenti: "The labour market has nevertheless seen the most dramatic changes in its history" (Commissione europea 2021). Secondo I'ILO (2021), la pandemia a livello globale ha causato la perdita di un numero di ore di lavoro quattro volte superiore a quelle perse durante la crisi del 2008. II bilancio finale sull'occupazione è stato ampiamente mitigato dal ricorso a forme di riduzione dell'orario di lavoro; molti di quelli che hanno perso il lavoro sono entrati in una condizione di inattività. Quindi, a dicembre 2020, il tasso di disoccupazione per la UE27 risulta superiore solo di 0,8 punti percentuali rispetto allo stesso mese del 2019.

La crisi economica innescata dalla pandemia sta colpendo in particolare i giovani. A dicembre 2020 il tasso di disoccupazione dei 15-24enni in UE27 si attesta al $17,7 \%$, segnando 2,7 punti percentuali in più rispetto allo stesso mese del 2019; per gli adulti 25-74enni nello stesso intervallo temporale, il tasso di disoccupazione è passato invece dal $5,8 \%$ al $6,5 \%$ (dati Eurostat). La fotografia per I'Italia mostra squilibri anche maggiori. Sulla popolazione dei 1564 enni, il tasso di disoccupazione su base tendenziale (dicembre 2020 su dicembre 2019) mostra una contrazione di 0,6 punti, a fronte di un aumento del tasso di inattività di 1,5 punti percentuali. Se invece si guarda al segmento dei giovani, nello stesso periodo la disoccupazione per i 15-24enni è aumentata di 1,3 punti percentuali, mentre il tasso di inattività è salito di 3 punti (Istat 2021).

Sono risultati che segnano una continuità con quanto registrato con le due crisi precedenti, la cosiddetta 'Grande Recessione' del 2008 e la successiva crisi del debito sovrano. Le due crisi hanno messo in evidenza la forte influenza che le fluttuazioni economiche esercitano sulla disoccupazione giovanile e quindi la maggiore vulnerabilità dei giovani rispetto agli adulti sul mercato del lavoro (Verick 2009; Piopiunik e Ryan 2012), soprattutto in quei Paesi che si confrontano con le maggiori difficoltà finanziarie ( $O^{\prime}$ Reilly et al. 2018). Si tratta di una conferma di quanto il dibattito fra gli esperti aveva evidenziato nell'esaminare il progressivo aumento della disoccupazione giovanile a partire dalla metà degli anni Settanta 
in un numero sempre più elevato di Paesi dell'area OECD: le trasformazioni delle economie e del lavoro intervenute a livello mondiale fanno dei giovani $i$ losers della globalizzazione (Blossfeld et al. 2005; Buchholz et al. 2009).

La mancanza di esperienza lavorativa che richiede un maggiore investimento in formazione da parte delle imprese e la minore capacità di ricerca di un lavoro legata anche alla scarsa disponibilità di reti di relazioni adeguate a facilitare l'inserimento vengono indicate come alcuni tra i fattori che annullano i vantaggi legati alla acquisizione di una forza lavoro più giovane e con un livello di istruzione e qualificazione mediamente più elevato rispetto ai lavoratori adulti, anche se non specifico rispetto alle esigenze della singola impresa (Bell e Blanchflower 2011); forti di un maggiore potere contrattuale e costrette a introdurre un livello sempre più alto di flessibilità per gestire l'incertezza legata alla globalizzazione, le imprese preferiscono inserire i giovani soprattutto attraverso forme di lavoro non standard, più flessibili e a costo inferiore (Blossfeld et al. 2005).

In generale la globalizzazione ha determinato un aumento delle disuguaglianze sociali, di cui hanno fatto le spese soprattutto i gruppi di popolazione più vulnerabili (Blossfeld et al. 2005; Buchholz et al. 2009; Quintini et al. 2007).

Le difficoltà di inserimento dei giovani nel mercato del lavoro si presentano con diversi gradi di gravità fra i vari Paesi, corroborando l'ipotesi che i diversi assetti istituzionali possono esercitare un peso nell'amplificare o meno il grado di vulnerabilità dei processi di transizione scuola/formazionelavoro (Caliendo e Schmidl 2016). Guardando agli assetti del mercato del lavoro, la presenza di un livello maggiore di precarizzazione e disoccupazione giovanile nei Paesi dell'Europa meridionale è stata messa in relazione con la maggiore sproporzione fra i benefici legati al possesso di una occupazione stabile rispetto alle forme non standard di lavoro; per questi contesti, il dualismo insider-outsider è stato identificato come uno dei fattori che può agire da cassa di risonanza (Blossfeld et al. 2005; Buchholz et al. 2009).

Anche le caratteristiche del sistema di istruzione e formazione sembrano giocare un ruolo nel favorire o meno la transizione scuola-lavoro. Ad esempio, i tassi di disoccupazione dei giovani dopo un anno dal completamento dei percorsi di istruzione sono molto più bassi in quei Paesi dove funzionano sistemi di apprendistato (Ryan 2001; Quintini et al. 2007; Hanushek et al. 2011). In questi stessi Paesi i dati mettono in evidenza che il prezzo pagato dai giovani per effetto delle due crisi del 2008 e 2011 è stato molto più contenuto.

In generale, le crisi precedenti hanno reso più difficile il passaggio dalla scuola al lavoro soprattutto per quei giovani che non hanno un background formativo adeguato alle esigenze del cambiamento strutturale e tecnologico, ma anche per quelli che non avrebbero avuto difficoltà in condizioni normali (Scarpetta et al. 2010). Per evitare che una difficile transizione crei 'cicatrici' permanenti sui percorsi di carriera individuali, alcuni Paesi hanno istituito o rafforzato il set di soggetti, strumenti e interventi finalizzati ad accompagnare e sostenere l'ingresso nel mercato del lavoro.

\section{Politiche attive del lavoro e formazione: il} contributo dei sistemi duali europei

All'indomani della Grande Recessione, i Paesi europei hanno cercato di fronteggiare gli squilibri nel mercato del lavoro facendo ampio ricorso alle politiche attive del lavoro (ALMP - Active Labour Market Policies) ${ }^{1}$, seguendo anche le indicazioni di organismi internazionali come OECD e UE che dagli anni Novanta ne avevano dichiarato l'efficacia per combattere la disoccupazione.

Rientrano nelle ALMP gli interventi che stimolano gli investimenti in capitale umano e l'occupazione (misure di supporto all'incontro domanda-offerta, incentivi all'assunzione, lavoro pubblico), che operano quindi sia sul piano della domanda che su quello dell'offerta di lavoro (Eichhorst e Konle-Seidl 2008; Bonoli 2010). In particolare l'attenzione allo sviluppo delle competenze individuali come strategia per fronteggiare la disoccupazione si sposa con il paradigma dell'investimento sociale (Hemerijck 2017), che punta a stimolare l'attivazione dei singoli, legando a questa la protezione sociale.

La letteratura ha ampiamente segnalato I'importanza dell'investimento nelle ALMP per contrastare la disoccupazione giovanile, anche

1 Teorizzate negli anni Cinquanta nell'ambito del cosiddetto 'Rehn-Meidner model', dal nome dei due economisti svedesi Gösta Rehn e Rudolf Meidner che svilupparono il modello. Per ulteriori informazioni si veda Erixon (2010). 
nelle fasi di crisi economica, soprattutto per evitare la disoccupazione di lunga durata o il ritorno nella inattività (Scarpetta et al. 2010; Caliendo e Schmidl 2016). Tuttavia in molti casi, all'indomani della grande recessione, il deficit accumulato dai Paesi non ha consentito un investimento forte sulle ALMP: le politiche di austerity hanno avuto maggiore presa sui decisori, orientando le scelte di policy verso l'incremento di misure condizionali (Barberis e Sergi 2016). Rimane infatti una questione aperta in letteratura quella di come conciliare i necessari investimenti sulle ALMP nei casi di ampio debito pubblico. Oltretutto, l'evidenza empirica ha mostrato che una spesa significativa in ALMP, da sola, non è sufficiente a contrastare una massiccia disoccupazione giovanile in presenza di una domanda di lavoro debole.

Tanto nella modellizzazione proposta da Bonoli (2010), quanto in quella che fa riferimento a Caliendo e Schmidl (2016), una parte delle politiche attive del lavoro è finalizzata al superamento dello skill mismatch e quindi guarda (anche) ai sistemi di formazione professionale come contesto nel quale inserire misure volte a promuovere lo sviluppo di competenze personali e professionali utili a sostenere l'occupabilità dei giovani. A tal fine, i sistemi di istruzione e formazione professionale sono stati stimolati a rinnovarsi per assicurare una maggiore coerenza con i fabbisogni espressi dalle imprese che cambiano ad un ritmo sempre più veloce inseguendo l'innovazione tecnologica.

Questo impegno al rinnovamento ha trovato un riferimento importante a livello europeo con l'avvio del processo di cooperazione volontaria in materia di istruzione e formazione (Processo di Copenhagen). L'obiettivo di "diventare l'economia basata sulla conoscenza più competitiva e dinamica del mondo, in grado di realizzare una crescita economica sostenibile con nuovi e migliori posti di lavoro e una maggiore coesione sociale" ${ }^{\prime 2} \mathrm{si}$ lega all'impegno a rafforzare i sistemi di istruzione e formazione per adeguarli ai bisogni dei sistemi produttivi e alle esigenze di promozione di una maggiore coesione sociale.

II percorso ormai pressoché ventennale di collaborazione a livello europeo ha progressivamente ampliato e dettagliato gli ambiti di cooperazione, individuando strumenti di supporto e relativi finanziamenti. Oggi, nel momento in cui si definiscono gli interventi europei per sostenere una ripresa competitiva e sostenibile, si sottolinea che I'Istruzione e formazione professionale (IFP) è “... fondamentale per conseguire gli obiettivi sociali ed economici dell'Unione europea" e dunque c'è una urgente necessità "di modernizzare e ampliare in modo significativo le politiche di IFP per renderle più inclusive, accessibili, resilienti, interessanti ed efficaci nel sostenere un'occupazione equa, lo sviluppo del capitale umano e la partecipazione attiva alla società"3.

Sotto il profilo delle policy, questa concezione dell'IFP si traduce nell'implementazione di sistemi di lifelong learning, in cui trova posto una offerta formativa ampia e diversificata, capace di dare risposta alle diverse esigenze delle persone nelle varie fasi della vita, professionale e non ${ }^{4}$. Nel perseguire una maggiore integrazione fra formazione e lavoro, il work based learning diventa uno strumento centrale, di cui si raccomanda l'inserimento in pressoché tutte le diverse offerte formative.

Fra gli strumenti di work based learning, un ruolo preminente è riconosciuto all'apprendistato. Il sistema duale di riferimento, ovvero il modello cui si ispirano quei Paesi che vogliono implementare lo strumento, è quello che opera in Germania e in Paesi limitrofi quali Svizzera e Austria: si tratta dei Paesi riconosciuti come apprenticeship countries (ILO 2012). Guardando alle serie storiche, questi Paesi mostrano i più bassi tassi di disoccupazione giovanile a livello europeo e di OECD e le loro politiche si sono dimostrate efficaci per assicurare ai giovani un buon inizio nel mercato del lavoro. In generale, è dimostrato che l'apprendistato è un percorso efficace per entrare nel mercato del lavoro e consente agli studenti con scarsi risultati di conseguire una qualifica. Anche durante una recessione economica e nelle prime fasi della ripresa, l'apprendistato può svolgere un ruolo fondamentale nel promuovere l'accesso al lavoro per i giovani (Scarpetta et al. 2010).

L'impulso alla diffusione dell'apprendistato

2 Consiglio europeo di Lisbona 23 e 24 marzo 2000 conclusioni della Presidenza, https://bit.ly/3m7E4TB.

3 Risoluzione del Parlamento europeo del 17 dicembre 2020 sulla raccomandazione del Consiglio relativa all'istruzione eformazione professionale (IFP) per la competitività sostenibile, l'equità sociale e la resilienza (2020/2767(RSP)), https://bit.ly/3zSSuvV.

4 Si veda nota 3. 
si è accompagnato allo sviluppo di un dibattito sulle sfide che tale strumento deve fronteggiare in relazione ai megatrend di cambiamento: terziarizzazione, globalizzazione, informatizzazione, automazione, calo demografico e invecchiamento della popolazione ${ }^{5}$. Sono processi - ancora in corso - che hanno investito tutti i Paesi, interessando i sistemi economici per poi sollecitare i sistemi del lavoro e del welfare.

Anche I'Italia ha inserito un intervento sull'apprendistato nel set di politiche attive per contrastare la disoccupazione giovanile. Prima con la riforma operata nel 2011 e poi con la revisione apportata con il D.Lgs. n. 81/2015 si è cercato di rivitalizzare l'istituto modificandone la connotazione e definendo forme di apprendistato collegate al conseguimento dei titoli di studio, progettate guardando all'esperienza di alcuni Paesi europei.

\section{Traiettorie di evoluzione di alcuni sistemi duali europei}

Al fine di fornire un contributo in merito alla implementazione in Italia delle forme di apprendistato collegate ai titoli di studio, l'Inapp ha promosso una attività di ricerca finanziata a valere sulle risorse del PON SPAO e finalizzata ad esaminare alcuni sistemi duali tra i più consolidati nel panorama europeo (D'Agostino e Vaccaro 2021). Analizzando in particolare le trasformazioni intervenute negli ultimi decenni, la ricerca ha indagato le traiettorie di evoluzione dei sistemi duali dei cosiddetti Paesi DACH - Germania, Austria, Svizzera - mettendoli a confronto con le trasformazioni intervenute invece in Francia e, soprattutto, in Italia, Paesi che tradizionalmente condividono un modello di formazione iniziale di tipo scolastico, a tempo pieno.

Tutti i Paesi analizzati nell'ambito della ricerca condividono un background culturale abbastanza omogeneo, costruito attraverso scambi frequenti, plurisecolari, di collaborazione e confronto che hanno favorito contaminazioni di cui restano tracce più o meno evidenti negli attuali sistemi di istruzione e formazione. La rete di relazioni e rapporti di forza/convenienza che lega stakeholder e soggetti istituzionali nei contesti specifici connota, invece, le differenze fra questi sistemi, caratterizzando diversamente gli assetti e le interconnessioni esistenti fra sistemi di istruzione e formazione, sistemi economici e del lavoro, sistemi di welfare.

In particolare, le interdipendenze funzionali fra formazione e lavoro definiscono diversi regimi di formazione delle competenze, identificati da Busemeyer e Trampusch (2012) secondo una matrice le cui determinanti sono legate all'impegno più o meno forte delle imprese nella formazione dei propri dipendenti da una parte e, dall'altra parte, al contributo dei soggetti istituzionali nel promuovere la formazione professionale. Germania, Austria e Svizzera si connotano come sistemi 'collettivisti' o 'corporativisti', in cui una grande partecipazione delle imprese (e dei loro soggetti di rappresentanza) si coniuga con un altrettanto forte impegno dei soggetti pubblici; la Francia è considerato, invece, un sistema statalista in cui al centro della formazione delle competenze è il settore pubblico.

Per i Paesi DACH, è il modello di corporativismo, ovvero di gestione del sistema duale attraverso il consenso, l'elemento che funge da motore e che assicura un impegno continuo per l'adattamento alle trasformazioni dell'economia e del lavoro e per il miglioramento dei risultati. Si tratta di un elemento che spesso sfugge o viene sottovalutato nel momento in cui si vuole avviare programmi di apprendistato mutuando l'esperienza tedesca (Eichhorst et al. 2015), come è avvenuto e sta ancora avvenendo in tanti altri Paesi in Europa - Italia compresa - nello sforzo di contrastare la disoccupazione giovanile. La gestione del sistema fortemente partecipata dalle organizzazioni di rappresentanza del lavoro è la precondizione per assicurare una attenzione continua alla qualità della formazione erogata, che rende il duale un'alternativa credibile ai percorsi di formazione generale, per i giovani e le loro famiglie (Eichhorst et al. 2015).

Anche i Paesi con un sistema duale forte e consolidato negli ultimi decenni hanno dovuto confrontarsi con gli effetti delle trasformazioni legate all'innovazione tecnologica, alla globalizzazione e terziarizzazione, all'evoluzione demografica e all'allungamento dei percorsi di scolarizzazione. E lo hanno fatto implementando misure di policy e riforme strutturali definite sempre attraverso

5 Su questo tema Cedefop e OECD hanno organizzato un Joint Simposium il 07 ottobre 2019. Tutti i materiali sono disponibili all'indirizzo https://bit.ly/39JnC6m. 
processi di concertazione, tenendo fermo l'impegno ad assicurare la qualità del sistema, intesa soprattutto come capacità di adeguarsi alle esigenze delle imprese in un contesto caratterizzato dal cambiamento. A giudicare dai risultati relativi alla partecipazione ai sistemi duali e dagli esiti in termini di successo formativo e occupazionale per gli apprendisti, è evidente che il sistema duale continua a svolgere nei Paesi DACH una funzione centrale per l'acquisizione di conoscenze e competenze generali e professionali per un'ampia quota di giovani ${ }^{6}$. In Germania, il sistema duale vanta una partecipazione di 1.328.964 apprendisti nel 2019, di cui 513.309 nuovi ingressi (Bibb 2020); nel 2018 tra i 18-34enni che hanno conseguito una qualifica nel sistema duale il tasso di disoccupazione era del 3,2\% (Solga et al. 2014). In Svizzera, 220.894 giovani risultano inseriti in un percorso di formazione iniziale nell'anno formativo 2018-19, quasi tutti in apprendistato $(90,6 \%)$; della coorte dei qualificati nel 2012, a tre anni e mezzo dalla fine del percorso solo l' $8 \%$ di quanti hanno conseguito un attestato federale di capacità (AFC) non lavora e non risulta in formazione (OFS 2018). Infine, in Austria nel 2019 il 39,5\% del totale dei quindicenni ha scelto un percorso di apprendistato ${ }^{7}$, mentre tra coloro che hanno conseguito una qualifica nel sistema duale il tasso di disoccupazione era del 3,5\% (Dornmayr e Löffler 2020).

In Francia l'apprendistato si caratterizza prevalentemente come percorso alternativo - la cosiddetta voie professionnelle - rispetto ai corsi a tempo pieno per l'acquisizione dei titoli del sistema di istruzione. Tutto il sistema di formazione professionale nell'ultimo decennio è stato attraversato da un profondo rinnovamento, che ne sta progressivamente modificando l'assetto. L'ultima riforma è stata approvata dal governo Macron nel $2018^{8}$, con un percorso di implementazione legato alla definizione di regolamentazioni attuative non ancora completato.

L'analisi delle traiettorie di evoluzione di tutti i sistemi europei di apprendistato presi in esame mette in risalto una dinamica di grande espansione dello strumento. Le trasformazioni strutturali dei sistemi economici hanno imposto un ampliamento in senso orizzontale, con la graduale estensione a nuovi profili e nuovi settori economici. Dal nucleo originario delle imprese artigiane del settore manifatturiero l'apprendistato ha progressivamente 'colonizzato' il terziario, ambito che storicamente ha promosso percorsi di costruzione delle professionalità centrati più spesso sulla formazione a tempo pieno (Thelen 2007).

Guardando, invece, alle qualificazioni acquisibili attraverso l'apprendistato, l'espansione dello strumento si è mossa in una direzione 'verticale'. Infatti, la transizione verso un'economia della conoscenza ha determinato una modifica dei fabbisogni di competenza espressi dalle imprese, a favore di figure professionali caratterizzate da una maggiore trasversalità, fondata su un livello più elevato di conoscenze e competenze di base che spesso deve coniugarsi con un set più ampio di competenze specialistiche. Ciò ha imposto a tutti $\mathrm{i}$ Paesi uno sforzo per il rinnovamento dei curricula per la formazione e uno spostamento del centro di gravità della formazione duale dal rilascio di qualifiche professionali post-obbligo di istruzione, collocabili al livello 3 dell'European Qualification Framework, a qualifiche di livello superiore, corrispondenti al livello 4 EQF (pari al diploma secondario superiore) o anche di livello 5, ovvero corrispondenti ai titoli dell'Istruzione tecnica superiore.

L'espansione in senso verticale ha prodotto una stratificazione interna dell'apprendistato, articolata su una riorganizzazione gerarchica legata all'occupazione di riferimento; il titolo di studio richiesto, l'attrattività in termini di chance di inserimento, la remunerazione attesa, la stabilità del lavoro, le possibilità di carriera, diventano elementi distintivi che orientano la domanda e la competizione per l'accesso ai vari percorsi di qualificazione.

Tale stratificazione è stata resa esplicita in alcuni Paesi attraverso modifiche strutturali al sistema duale che hanno visto l'introduzione di percorsi di durata differenziata: in Germania nel 2003 vengono introdotti percorsi di durata biennale - mentre generalmente un apprendistato dura da 36 a 42 mesi; in Svizzera nel 2002 viene introdotto il certificato federale CFP acquisibile al termine di un percorso biennale - distinto dal percorso per l'acquisizione dell'AFC per il quale occorrono 3-4 anni.

6 Per maggiori informazioni si rimanda a D’Agostino e Vaccaro (2021).

7 Dati tratti dal sito della Camera dell'Economia, https://bit.ly/3zPCfQ9.

8 L. n.2018-771 del 5 settembre 2018, Pour la liberté de choisir sono avenir professionnel. 
In Francia l'espansione dell'apprendistato verso i livelli più elevati del sistema di istruzione avviene tra la fine degli anni Ottanta e i primi anni Novanta, attraverso l'ampliamento del ventaglio dei diplomi conseguibili in apprendistato, prima ai titoli dell'istruzione secondaria superiore e, successivamente, ai titoli del sistema terziario. Per rivitalizzare l'apprendistato si punta dunque ad un allargamento della sua offerta formativa e a un innalzamento del livello dei titoli conseguibili, sostituendo alla gerarchia informale dei mestieri una gerarchia formale dei diplomi alla stregua del sistema scolastico (Moreau 2008).

Negli stessi anni anche gli altri Paesi mettono in discussione le barriere esistenti tra i vari livelli del sistema educativo, il cosiddetto "scisma educativo" (Baethge 2006), aumentando il grado di permeabilità. Ad esempio, in Svizzera nel 1993 viene introdotta la maturità professionale che consente l'accesso alle Università di Scienze applicate a conclusione del percorso duale; successivamente (2002), vengono attivate le Passerellen per l'ingresso all'università. In Austria nel 2008 viene introdotto il programma Lehre mit Matura che consente a tutti gli apprendisti di conseguire parallelamente la maturità professionale per accedere poi agli studi terziari.

Le innovazioni apportate ai sistemi duali dei Paesi analizzati raggiungono l'obiettivo di attrarre nel sistema una quota crescente di giovani che aspirano a collocarsi nei livelli professionali più alti determinando però il restringimento delle opportunità disponibili per i giovani con un percorso scolastico meno brillante o con un'origine sociale popolare. I dati raccolti mettono in evidenza le difficoltà dei più vulnerabili a fronte della trasformazione dei sistemi di apprendistato in una prospettiva che insegue l'evoluzione dei sistemi economici verso l'innalzamento dei livelli di qualificazione ${ }^{9}$. In Germania, ad esempio, all'interno del sistema duale, i percorsi di coloro che hanno bassi livelli di competenza sono più accidentati: il tasso di risoluzione anticipata del contratto è del $39,3 \%$ per gli apprendisti senza qualificazione di scuola secondaria superiore e del $15,4 \%$ tra coloro che dispongono di un titolo di accesso all'università. I primi presentano anche un tasso di successo agli esami finali più basso (84\%) rispetto ai secondi
(97,9\%) (BMBF 2020). In Francia, se fino agli anni Ottanta la provenienza da una famiglia operaia era di gran lunga più comune tra gli apprendisti che tra gli studenti delle scuole professionali, dagli anni Novanta i due gruppi riducono la loro distanza e gradualmente l'origine sociale degli apprendisti diventa meno popolare di quella degli studenti a tempo pieno. Negli anni 2000, il 41\% degli apprendisti che si prepara a conseguire il primo livello di qualificazione (CAP - Certificato di attitudine professionale) ha il padre operaio (erano il 59\% nel 1978) contro il $45 \%$ degli studenti; tra gli apprendisti che seguono un percorso di istruzione terziaria, solo il $19 \%$ proviene da una famiglia operaia, mentre gli studenti con la stessa origine sono il 21\% (Kergoat 2015).

Non solo i giovani con percorsi precedenti più accidentati e/o provenienti da contesti socioeconomici più svantaggiati, ma anche quelli che hanno un'esperienza di migrazione incontrano maggiori difficoltà a trovare un posto in apprendistato, ottengono risultati medi significativamente peggiori rispetto ai non migranti e quindi fanno più fatica ad inserirsi stabilmente nel mondo del lavoro (Granato e Ulrich 2017). In Austria, ad esempio, nell'anno scolastico 2018-19 la proporzione di stranieri in prima elementare era del $20 \%$, mentre nel sistema duale si fermava al $13,7 \%$; la percentuale di coloro che non utilizzano il tedesco come lingua principale di comunicazione costituiva il $31 \%$ nelle scuole elementari, nel duale arrivava al 18,2\% (Dornmayr e Löffler 2020). Il tasso di abbandono scolastico è pari in media al 7,3\%, ma tre volte superiore per i nati all'estero rispetto ai nativi. In Svizzera la quota di apprendisti stranieri nell'anno 2018-2019 è del 21,6\% sul totale degli utenti e in generale questi giovani devono impegnarsi molto per trovare un'azienda che li accolga: secondo i dati del Barometro della transizione di agosto 2019, devono presentare in media 25,7 candidature, mentre gli svizzeri solo 7,2.

\section{L'apprendistato in Italia: un modello sui generis}

L'apprendistato in Italia è sempre stato concepito come un istituto con una valenza prevalentemente occupazionale (Carinci 2012); di conseguenza la decisione dei datori di lavoro di utilizzare questa

9 Cfr. nota 6 . 
tipologia di contratto è stata spesso dettata da considerazioni legate al costo del lavoro e al risparmio conseguito rispetto ad altre forme contrattuali. La componente formativa dell'apprendistato, che rappresenta o dovrebbe rappresentare la sua ragione costitutiva, è stata vissuta, quindi, in larga misura, come un elemento estraneo, se non come una inutile complicazione.

Questa visione dell'apprendistato è stata in un certo senso assecondata dalla gran parte dei provvedimenti adottati nel corso degli anni per promuovere la diffusione del dispositivo. Le misure di policy hanno infatti agito soprattutto sulla leva degli incentivi alle imprese, sollecitando l'attenzione alla convenienza economica dello strumento e favorendo la competizione con altre forme contrattuali o modalità di inserimento al lavoro. Nello stesso tempo si è intervenuti sulla componente formativa dell'apprendistato sia riducendone progressivamente il peso in termini di ore di formazione pubblica erogata, sia indebolendone l'obbligatorietà.

Non è quindi un caso se le caratteristiche prevalenti del modello italiano di apprendistato continuino a identificarsi con una delle tre tipologie $^{10}$ che compongono il sistema, quella cosiddetta professionalizzante o di secondo livello che, nel 2017, rappresentava il 97\% del numero medio dei rapporti di lavoro in apprendistato (Inapp 2019a). Questa tipologia non porta ad acquisire un titolo di studio, essendo finalizzata esclusivamente al conseguimento di una qualifica professionale contrattualmente prevista; è quindi la forma di apprendistato meno integrata nel sistema di istruzione e formazione (Tiraboschi 2016) e la più lontana dai sistemi duali europei.

La formazione nell'apprendistato professionalizzante ha una componente pubblica, a cura delle Regioni e delle Province autonome, finalizzata allo sviluppo delle competenze di base e trasversali. Questa componente è stata ridimensionata sia attraverso una riduzione delle ore di formazione erogate, il cui ammontare complessivo non supera attualmente le 120 ore, sia legando l'obbligo di erogazione alla disponibilità sul territorio di un'offerta formativa effettivamente fruibile da parte delle imprese. La debolezza della formazione pubblica è confermata dai dati: nel 2017 ne ha beneficiato a livello nazionale un apprendista su tre, ma nel Mezzogiorno solo il 4\% (Inapp 2019a). È prevista anche una formazione tecnico-professionale a cura delle imprese che varia nella durata e nell'articolazione secondo le indicazioni della contrattazione collettiva, ma mediamente lo standard previsto è limitato a 80 ore.

Al contrario dell'apprendistato professionalizzante, la tipologia di primo livello consente di conseguire un titolo di studio di istruzione secondaria superiore (qualifica triennale o diploma professionale dei percorsi di istruzione e formazione professionale regionali, certificato di specializzazione tecnica superiore e diploma di scuola secondaria superiore), mentre la tipologia di terzo livello è destinata alla formazione universitaria (master, lauree triennali e specialistiche, dottorati di ricerca), all'attività di ricerca e al praticantato per l'accesso alle professioni ordinistiche. Queste tipologie cosiddette formative e quindi più vicine ai sistemi duali europei hanno sempre avuto in Italia un peso residuale, tra il $3 \%$ e il $4 \%$ dei rapporti di lavoro complessivi in apprendistato: nel 2017 il numero medio di rapporti di lavoro in apprendistato di primo e terzo livello è di poco superiore a 10.000 e risulta in calo di circa lo 0,3\% rispetto all'anno precedente (Inapp 2019a).

L'analisi dei dati disponibili sulla diffusione dell'apprendistato ci restituisce un'immagine di questo strumento non particolarmente positiva. Le sue caratteristiche principali sono infatti la prociclicità, la volatilità e la ineguale distribuzione tra i territori e i settori economici.

L'apprendistato risulta fortemente condizionato dalla congiuntura economica. Infatti, durante gli anni della Grande Recessione, questo istituto contrattuale ha subito un continuo calo, passando dai 619.334 rapporti medi di lavoro del 2008 ai 374.253 del 2016, con una riduzione complessiva di circa il $40 \%$. Nel 2017, a seguito di una timida ripresa, si è registrata una inversione di tendenza che è proseguita sino al 2019 quando il numero medio dei rapporti di

10 Secondo il capo V del D.Lgs. n. 81/2015, il contratto di apprendistato si articola nelle seguenti tipologie: a) apprendistato per la qualifica e il diploma professionale, il diploma di istruzione secondaria superiore e il certificato di specializzazione tecnica superiore; b) apprendistato professionalizzante; c) apprendistato di alta formazione e ricerca. La prima tipologia è rivolta ai giovani che hanno compiuto i 15 anni di età e fino al compimento dei 25 anni; la seconda e la terza interessano i giovani di età compresa tra i 18 e i 29 anni. 
lavoro in apprendistato è arrivato a 549.342, senza tuttavia raggiungere i livelli del $2008^{11}$. Con la crisi determinata dalla pandemia da Covid-19 si è avviata una nuova fase di contrazione dell'apprendistato già nel primo trimestre del 2020 che è proseguita nel periodo successivo facendo registrare nel terzo trimestre una diminuzione tendenziale pari al $12,7 \%$ rispetto allo stesso periodo del 2019 (Ministero del Lavoro e delle politiche sociali 2020). II carattere pro-ciclico dell'apprendistato mostra la scarsa resilienza di questo strumento, mettendo in discussione una delle principali virtù che gli sono riconosciute: la capacità di proteggere i giovani dal rischio della disoccupazione. L'istituto conserva, inoltre, un carattere residuale sul mercato del lavoro rispetto ad altre tipologie contrattuali: dal 2009 al 2017, la percentuale di attivazioni con contratto di apprendistato è rimasta attestata tra il $14 \%$ e il $15 \%$ delle attivazioni totali ${ }^{12}$.

I dati sulla diffusione dell'apprendistato a livello nazionale sono il risultato di situazioni regionali molto diverse tra loro. Se guardiamo per ragioni di sintesi alle macroaree regionali, risulta evidente la ineguale distribuzione dei contratti: nel Nord Italia si concentra, infatti, più della metà del numero medio dei rapporti di lavoro in apprendistato, mentre il Mezzogiorno è rimasto costantemente al di sotto del $20 \%$. Differenze rilevanti si registrano anche a livello settoriale: i comparti del Commercio e dei Servizi di alloggio e ristorazione raccolgono nel 2017 il $38 \%$ dell'occupazione in apprendistato, con un incremento di sei punti percentuali rispetto al 2008 (Inapp 2019a).

La concentrazione dell'apprendistato in comparti caratterizzati dalla prevalenza di occupazioni per le quali non è richiesto un elevato livello di competenze, insieme alle caratteristiche dei lavoratori (giovani adulti, tra i 18 e i 24 anni, ma spesso e sempre di più anche tra i 25-29enni, mentre la presenza di minori non supera lo $0,6 \%$ ), ci forniscono la fotografia di uno strumento concepito prevalentemente come contratto per l'inserimento al lavoro in occupazioni a basso livello di qualificazione, i cui contenuti fondamentali si possano apprendere semplicemente tramite l'affiancamento e l'osservazione del collega con più esperienza (Cefalo 2015).
Infine, i dati sulla durata dei contratti di apprendistato evidenziano la volatilità che contraddistingue questo strumento: nel 2015 più del $45 \%$ di apprendisti ha sottoscritto un contratto che è cessato entro un anno dalla stipula (Inapp 2019a). Si tratta quindi di un rapporto di lavoro fortemente discontinuo che viene percepito come temporaneo e occasionale tanto dalle imprese quanto dai giovani, visto che le dimissioni volontarie sono la prima causa di cessazione del rapporto di lavoro (D'Agostino e Vaccaro 2019).

\section{Le traiettorie di evoluzione dell'apprendistato in Italia}

Con la più recente riforma dell'apprendistato, avvenuta nel 2015 con il D.Lgs. n. 81, si è cercato di imprimere una svolta rispetto alla situazione descritta, ponendo le basi per la costruzione di una 'via italiana al sistema duale', che si ispira alle migliori pratiche europee.

Per conseguire questo obiettivo il Ministero del Lavoro e le Regioni hanno deciso di concentrarsi sulla promozione dell'apprendistato di primo livello, insieme all'alternanza scuola-lavoro e all'impresa formativa simulata, all'interno della filiera dell'Istruzione e Formazione professionale (leFP).

Nel 2015 è stata promossa una iniziativa a carattere sperimentale che avrebbe dovuto consentire l'attivazione di percorsi di apprendistato per 20.000 giovani, favorendo il loro inserimento stabile nel mondo del lavoro e riducendo nello stesso tempo la dispersione scolastica e formativa e il numero dei giovani non impegnati né nel lavoro né nella formazione.

I dati sui risultati della sperimentazione conclusa nel 2017 non sono incoraggianti: gli apprendisti inseriti nei percorsi di leFP per il conseguimento della qualifica e del diploma professionale sono 2.082 che si aggiungono ai 3.389 partecipanti ai percorsi formativi di leFP al di fuori della sperimentazione, per un totale di 5.471 apprendisti (Inapp 2019a).

Ma soprattutto questi dati confermano una linea di tendenza consolidata alla concentrazione degli apprendisti di primo livello in un numero molto limitato di territori: la Provincia autonoma di Bolzano, dove si trova il 60\% degli apprendisti e dove

11 Dati Inps - Osservatorio sui lavoratori dipendenti.

12 Elaborazioni Osservatorio statistico Consulenti del lavoro su micro-dati CICO (Comunicazioni obbligatorie). 
il modello di apprendistato è molto simile al sistema duale tedesco e la Regione Lombardia (con il 30\% di apprendisti) che da tempo investe nella costruzione di un apprendistato duale. Al Sud invece gli apprendisti di primo livello sono sostanzialmente assenti.

Peraltro, la scelta di incardinare l'apprendistato duale nella leFP non ha favorito una diffusione più omogenea del duale sul territorio nazionale. La leFP è, infatti, un canale che si è sviluppato in modo fortemente diseguale in esito alle scelte sull'organizzazione dell'offerta formativa e sulle risorse da impiegare adottate dalle singole Regioni che esercitano una competenza esclusiva in questo ambito. II risultato di queste scelte è che nel Nord del Paese si concentra, nell'anno formativo 2016-2017, poco meno dell' $80 \%$ del numero di iscritti ai percorsi di leFP erogati a tempo pieno, di cui quasi il $60 \% \mathrm{si}$ trova in Lombardia, Veneto e Piemonte, mentre il Mezzogiorno conta poco meno del $12 \%$ degli iscritti (Inapp 2019b).

La scelta di promuovere l'apprendistato di primo livello nel canale della leFP ha certamente favorito le Regioni settentrionali che da anni investono in questa filiera formativa, offrendo l'occasione per rafforzare i legami con il tessuto produttivo locale e per sperimentare strategie innovative dirette a rendere più flessibile l'organizzazione didattica e a personalizzare i percorsi formativi (Gotti e Piano 2016).

Per sostenere la diffusione del duale su tutto il territorio nazionale sarebbe stato tuttavia indispensabile attivare le misure necessarie a superare le fragilità dei sistemi regionali di leFP, le cui cause sono note da tempo: mancata conformità ai livelli essenziali delle prestazioni del secondo ciclo del sistema educativo (D.Lgs. n. 226/2005); risorse finanziarie non continuative e insufficienti a garantire la copertura della domanda di formazione dei giovani e delle loro famiglie; limitata corrispondenza tra l'offerta formativa e i fabbisogni formativi e professionali espressi dal sistema produttivo; assenza di standard di qualità applicabili su tutto il territorio nazionale; scarso investimento nelle risorse umane e strumentali (Associazione Treellle e Fondazione per la Scuola 2016).

La riforma dell'istruzione professionale avviata nel 2015 , in concomitanza con la riforma dell'apprendistato, avrebbe potuto rappresentare l'occasione per rilanciare su nuove basi la filiera della leFP, ma a questo scopo si sarebbe dovuta formare una precisa volontà politica da parte sia del Governo, sia delle Regioni (Longhi 2017) che, purtroppo, non si è manifestata. L'intervento legislativo si è infatti limitato a fissare le modalità di raccordo tra il sistema dell'istruzione professionale e quello dell'istruzione e formazione professionale e i passaggi dall'uno all'altro, senza però regolare la leFP. Di conseguenza, in questo canale, l'offerta formativa continua a soffrire la mancanza di una programmazione pluriennale in grado di garantire un'offerta formativa ampia e stabile in tutto il Paese; ma, soprattutto, il canale della leFP regionale rimane privo di un adeguato sistema per l'accertamento del rispetto dei livelli essenziali delle prestazioni e di un modello unitario di 'costi standard', indispensabile per garantire ovunque oggettivi criteri di qualità, adeguatezza ed efficienza per l'offerta formativa (Zagardo 2019).

La decisione di mantenere lo status quo è indicativa della scarsa importanza attribuita in Italia alla formazione professionale che, infatti, non viene considerata come un servizio pubblico ad accesso universale, da assicurare a parità di condizioni e di trattamento (Salerno 2020). Si tratta di una pericolosa sottovalutazione che rischia di lasciare il nostro Paese privo di un importante strumento per supportare la ripresa dopo la crisi determinata dalla pandemia da Covid-19 e per rafforzare la capacità di resilienza sia degli individui che dei territori.

\section{Conclusioni}

I Paesi europei esaminati, sia quelli con sistemi duali che vantano una lunga tradizione (Germania, Austria, Svizzera), sia la Francia il cui sistema duale è meno consolidato, guardano all'apprendistato come a un'efficace misura di politica attiva per facilitare l'ingresso nel mercato del lavoro dei giovani e come una risposta utile al fabbisogno di competenze a lungo termine delle imprese (Cedefop 2021); nel contempo assegnano a questo dispositivo una funzione di inclusione sociale nei confronti, in particolare, dei giovani più vulnerabili.

In questi Paesi si è quindi deciso di intervenire con specifiche misure di policy o con riforme di vasta portata per elevare la qualità dei sistemi duali, la cui capacità di attrazione nei confronti dei giovani e delle imprese si era appannata nel corso degli anni sotto l'effetto di alcuni megatrend globali, tra i quali l'innovazione tecnologica, i cambiamenti demografici, la terziarizzazione dell'economia e la globalizzazione. Gli interventi promossi per superare queste difficoltà seguono traiettorie di evoluzione 
che, pur mantenendo le peculiarità di ciascun sistema, presentano alcuni punti di caduta comuni.

L'analisi dei punti di convergenza consente di individuare sia alcuni aspetti positivi nelle traiettorie di evoluzione dei sistemi duali (in termini di aumento della qualità dell'apprendimento e di miglioramento della percezione che le imprese, i giovani e le loro famiglie hanno dell'apprendistato), sia talune criticità che afferiscono in particolare alla sempre più limitata capacità di inclusione che questo dispositivo è in grado di esercitare nei confronti dei giovani socialmente svantaggiati. A partire da questa analisi è possibile svolgere alcune prime riflessioni sulle strategie adottate dall'Italia per promuovere la costruzione di una propria via al sistema duale e sulle misure che il Paese potrebbe attivare per consentire all'apprendistato di svolgere una funzione di contrasto alla crisi dell'occupazione giovanile aggravata dalla pandemia da Covid-19.

Una prima convergenza tra i sistemi duali dei Paesi europei esaminati si registra in relazione alla loro progressiva integrazione nel sistema educativo generale e al riposizionamento, in forme differenziate e nuove ibridazioni, verso l'istruzione superiore non accademica e l'istruzione terziaria. Tale riposizionamento ha permesso ai sistemi duali di esercitare una attrazione crescente nei confronti dei giovani con più elevati livelli di qualificazione, il cui numero era nel frattempo cresciuto a causa dell'innalzamento generalizzato dei livelli di istruzione, e di rispondere alle mutate esigenze delle imprese.

Guardando a queste linee di evoluzione, I'Italia dovrebbe essere spinta, da un lato, a superare la separazione e la gerarchizzazione delle filiere dell'istruzione e formazione del ciclo secondario superiore, dando maggiore spazio in tutti i percorsi allo sviluppo delle competenze di base e generali e alle competenze trasversali e, dall'altro, a garantire, su tutto il territorio nazionale, la 'verticalizzazione' delle filiere formative professionalizzanti, attraverso la costruzione di un'offerta formativa più completa che consenta il passaggio all'istruzione terziaria secondo due modalità: i corsi per la qualifica e il diploma professionale che danno accesso all'Istruzione e formazione tecnica superiore (IFTS) e, per questa via, agli ITS (Istituti tecnici superiori); la partecipazione ad un quinto anno integrativo che permette l'ingresso all'università.

Una seconda linea di convergenza tra i sistemi duali europei riguarda le molteplici misure di policy attivate per migliorare la qualità dell'offerta formativa e dei processi di apprendimento. Per I'Italia, la scelta di puntare sulla qualità della formazione dovrebbe passare per il preventivo rilancio su nuove basi della filiera della leFP che si presenta fortemente differenziata per quantità e qualità dell'offerta formativa sul territorio nazionale e risulta pressoché assente proprio nelle aree del Paese, a partire dal Mezzogiorno, dove più evidenti sono i fenomeni di marginalità educativa e le difficoltà di transizione al lavoro dei giovani. È dunque necessario intervenire sulle debolezze di questo segmento formativo che continua a soffrire la mancanza di una programmazione pluriennale e di un sostegno finanziario adeguati a garantire continuità nell'attivazione di percorsi di apprendistato duale.

Per promuovere la qualità dell'apprendimento si dovrebbe, inoltre, evitare di scaricare sulle istituzioni formative l'intero onere della complessa costruzione dei percorsi di formazione duale. A questo scopo è necessario, in primo luogo, definire degli standard formativi analitici e dei curricula specifici per l'apprendistato che consentano di collegare formalmente le qualifiche conferite dai titoli di studio (qualifiche o diplomi di IFP, diplomi di istruzione tecnica o professionale) e le qualificazioni professionali (Cedefop 2017). Attraverso la costruzione di un framework unitario e condiviso con le Parti sociali, che integri a monte gli obiettivi formativi scolastici, quelli aziendali e l'esperienza lavorativa, si può, infatti, assicurare l'occupabilità a lungo termine dei discenti, supportata da competenze professionali non specifiche dell'impresa e da forti competenze trasversali, che permettano loro di trovare un impiego al di là di specifiche aziende e settori (Cedefop 2021).

Nello stesso tempo, lo sforzo compiuto dalle istituzioni formative nella costruzione dei percorsi di formazione duale dovrebbe essere sostenuto sia mediante l'adozione di misure di policy dirette a rafforzare le reti e i partenariati locali con le imprese, le istituzioni territoriali, le Parti sociali, i Centri per l'impiego e altri attori rilevanti a livello locale, sia attraverso interventi finalizzati all'attivazione di servizi di analisi dei bisogni di formazione e professionali, di accoglienza, di orientamento e di accompagnamento dell'utenza, di validazione delle competenze acquisite e di formazione degli operatori. 
Per migliorare la qualità dell'apprendistato, i Paesi europei esaminati hanno agito anche sul potenziamento della governance multilivello dei sistemi duali, attraverso la costruzione di sedi stabili e strutturate di confronto e condivisione, la stipula di accordi di collaborazione con le Parti sociali, il rafforzamento delle attività di analisi e di monitoraggio delle misure di policy attuate e di anticipazione dei fabbisogni formativi e professionali e la formazione dei soggetti coinvolti a vario titolo nel funzionamento del sistema duale.

Anche in Italia il rilancio dell'apprendistato richiederebbe un potenziamento della governance multilivello, da realizzare, sia attraverso la costituzione di un organismo di coordinamento nazionale che garantisca una gestione unitaria del sistema duale superando le tradizionali separazioni tra le filiere formative regionali e statali e favorendo l'attivazione di un processo di programmazione unificato anche a livello locale (Cedefop 2017), sia rafforzando il ruolo di coordinamento e di indirizzo dell'Amministrazione centrale e promuovendo un maggiore coinvolgimento delle Parti sociali, in particolare per l'analisi dei fabbisogni professionali e formativi delle imprese, per la costruzione dei repertori delle qualificazioni professionali e nelle attività di supporto ai lavoratori e alle imprese.

La terza e ultima linea di convergenza concerne la progressiva riduzione della capacità inclusiva dei sistemi duali. Nei Paesi europei esaminati si è determinata una graduale trasformazione nei rapporti tra sistema duale e sistema scolastico. Sino agli anni Settanta la competizione sul mercato dell'apprendistato per i posti e le aziende migliori non si giocava esclusivamente sui risultati conseguiti nel precedente percorso di studi e sul prestigio delle scuole frequentate; di conseguenza l'apprendistato, pur non svolgendo una funzione propriamente emancipatrice, era comunque in grado di offrire anche ai giovani in situazione di svantaggio l'opportunità di conseguire una qualifica con un valore riconosciuto e quindi la possibilità di un buon inserimento nel mercato del lavoro. A partire dagli anni Ottanta, la preselezione effettuata dal sistema scolastico assume progressivamente una maggiore importanza per l'ingresso in apprendistato e per il successivo posizionamento dei giovani nel mercato del lavoro, con un effetto negativo sulle traiettorie di coloro che sono privi di titoli di studio o sono in possesso di basse qualificazioni e dei giovani con precedenti percorsi di istruzione accidentati. Inoltre, i sistemi duali tendono a sottorappresentare sia $\mathrm{i}$ giovani con background migratorio, sia le donne; entrambi questi gruppi incontrano maggiori difficoltà a trovare un posto in apprendistato e spesso rimangono confinati in settori ed imprese che offrono prospettive di inserimento nel mercato del lavoro più difficili e in occupazioni più precarie $\mathrm{e}$ meno retribuite.

L'aumento delle disuguaglianze educative e nel mercato del lavoro non riguarda, dunque, soltanto I'Italia, ma nel nostro Paese questa criticità si presenta in forma particolarmente acuta, specialmente nelle regioni del Mezzogiorno, dove la dispersione scolastica e i giovani che non studiano e non lavorano raggiungono punte superiori rispettivamente al 20\% (Istat 2020) e al 35\% (Istat 2019).

II contrasto a questi fenomeni rappresenta quindi un obiettivo prioritario per I'Italia. II suo perseguimento però difficilmente può essere affidato all'apprendistato duale senza intervenire preliminarmente per correggere gli effetti della riproduzione delle disuguaglianze sociali prodotti dalla scuola e gli effetti di amplificazione di tali disparità che si registrano nel mercato del lavoro. 


\section{Bibliografia}

Associazione Treellle, Fondazione per la Scuola (2016), Accendere i fari sull'Istruzione e Formazione professionale (IFP-VET), Quaderno n.12, Genova, Associazione Treellle <https://bit.ly/3APw3ce>

Baethge M. (2006), Das deutsche Bildungs-Schisma. Welche Probleme ein vorindustrielles Bildungssystem In Einer nachindustriellen Gesellschaft hat, SOFI-Mitteilungen, n.34, pp.13-27<https://bit.ly/3zOqjhu>

Barberis E., Sergi V. (2016), Politiche attive per il mercato del lavoro nella crisi. Il quadro europeo e il caso italiano, Argomenti, n.5, pp.5-28<https://bit.ly/3zSg6AC>

Bell D., Blanchflower D. (2011), Young people and the Great Recession, Oxford Review of Economic Policy, 27, n.2, pp.241-267

Bibb- Bundesinstitut für Berufsbildung (eds.) (2020), Datenreport zum Berufsbildungsbericht 2020. Informationen und Analysen zur Entwicklung der beruflichen Bildung, Bonn, Bundesinstitut für Berufsbildung <https://bit.ly/3zTwINL>

Blossfeld H.P., Klijzing E., Mills M., Kurz K. (eds.) (2005), Globalization, Uncertainty and Youth in Society, London and New York, Routledge BMBF- Bundesministerium für Bildung und Forschung (2020), Berufsbildungsbericht 2020, Bonn, BMBF <https://bit.ly/3m1nWmG>

Bonoli G. (2010), The political economy of active labour-market policy, Working Papers on the Reconciliation of Work and Welfare in Europe n.1, Edinburgh, RECWOWE Publication <https://bit.ly/2Zvtcaw>

Buchholz S., Hofäcker D., Mills M., Blossfeld H.P., Kurz K., Hofmeister H. (2009), Life Courses in the Globalization Process. The Development of Social Inequalities in Modern Societies, European Sociological Review, 25, n.1, pp.53-71

Busemeyer M.R., Trampusch C. (2012), The comparative political economy of collective skill Formation, in Busemeyer M.R., Trampusch C. (eds.), The political economy of collective skill formation, Oxford, Oxford University Press, pp.3-40

Caliendo M., Schmidl R. (2016), Youth unemployment and active labor market policies in Europe, IZA Journal of Labor Policy, 5, n.1, pp.1-30<https://bit.ly/3uidPxw>

Carinci F. (2012), E tu lavorerai come apprendista (L'apprendistato da contratto "speciale" a contratto "quasi-unico"), Working Paper CSDLE "Massimo D’Antona” n.145, Catania, CSDLE <https://bit.ly/3CTyX07>

Cedefop (2021), Apprendistato. Una pillola per ogni male?, Nota informativa, marzo, Salonicco, Cedefop <https://bit.ly/2Wr60t0> Cedefop (2017), Apprenticeship review: Italy. Building education and training opportunities through apprenticeships. Thematic Country Reviews, Luxembourg, Publications Office of the European Union <https://bit.ly/3m9omYf>

Cefalo R. (2015), Alla ricerca di una cultura dell'apprendistato in Italia, Percorsi di Secondo Welfare, $1^{\circ}$ dicembre <https://bit.ly/39MAkBc> Commissione europea (2021), European Economic Forecast. Winter report (interim), European Economy Institutional Paper n.144, Luxembourg, Publications Office of the European Union <https://bit.ly/3m3E47m>

D’Agostino S., Vaccaro S. (2021), Traiettorie di evoluzione dei sistemi duali. Riflessioni per I'Italia, Roma, Inapp

D’Agostino S., Vaccaro S. (2019), Apprendistato e qualità: un binomio possibile?, Scuola Democratica. Learning for democracy, n.2, pp.431-443

Dornmayr H., Löffler R. (2020), Bericht zur Situation der Jugendbeschäftigung und Lehrlingsausbildung in Österreich. 20182019, Wien, Forschungsbericht von ibw und öibf im Auftrag des BMDW

Eichhorst W., Rodríguez-Planas N., Schmidl R., Zimmermann K.F. (2015), A road map to vocational education and training in industrialized countries, Industrial and Labor Relations Review, 68, n.2, pp.314-337

Eichhorst W., Konle-Seidl R. (2008), Contingent convergence. A comparative analysis of activation policies, IZA Discussion Paper n.3905, Bonn, IZA

Erixon L. (2010), The Rehn-Meidner Model in Sweden. Its Rise, Challenges and Survival, Journal of Economic Issues, 44, n.3, pp.677-715

Gotti E., Piano R. (2016), Il sistema duale nell'ambito dell'Istruzione e Formazione Professionale. L'avvio di una sperimentazione nazionale, Rassegna Cnos, 32, n.1, pp.107-123

Granato M., Ulrich J.G. (2017), L'alternance, une voie de réussite pour tous? L'impact de l'origine ethnique en Allemagne, Formation Emploi, n.139, pp.119-146

Hanushek E.A., Woessmann L., Zhang L. (2011), General education, vocational education, and labor-market outcomes over the life-cycle, IZA Discussion Paper n.6083, Bonn, IZA

Hemerijck A. (ed.) (2017), The uses of social Investment, Oxford, Oxford University Press

ILO (2021), ILO Monitor: COVID-19 and the world of work. Seventh edition. Updated estimates and analysis, 25 January, Genève, ILO <https://bit.ly/2Y5pMv1>

ILO (2012), Overview of apprenticeship systems and issues. ILO contribution to the G20 Task Force on Employment, November, Genève, ILO

Inapp (2019a), L'apprendistato tra continuità e innovazione. XVIII Rapporto di monitoraggio. Executive Summary, Roma, Inapp $<$ https://bit.ly/3EUXsMe>

Inapp (2019b), Rapporto annuale sulla sperimentazione del sistema duale nella leFP. A.f. 2016-2017, Roma, Inapp <https://bit.ly/2YbliTl>

132 L'apprendistato in Italia: una misura di politica attiva ancora poco valorizzata | D'Agostino, Vaccaro 
Istat (2021), Occupati e disoccupati. Dati provvisori, Statistiche Flash, $1^{\circ}$ febbraio, Roma, Istat <https://bit.ly/3FOMHYN> Istat (2020), Rapporto annuale 2020. La situazione del Paese, Roma, Istat <https://bit.ly/2Y1ISCF>

Istat (2019), Rapporto SDGs 2019. Informazioni statistiche per l'Agenda 2030 in Italia, Roma, Istat <https://bit.ly/3zQLXSs>

Kergoat P. (2015), Trouver et tenir sa place. Les apprenti(e)s et le travail, L'orientation scolaire et professionnelle, 44, n.2, pp.1-22 Longhi G. (2017), Addentrandosi nelle pratiche sul duale su scala nazionale, Skill. Teorie ed esperienze sulla formazione, n.4, pp.4-9 Ministero del Lavoro e delle politiche sociali (2020), Nota Comunicazioni Obbligatorie III trimestre 2020, n.35, dicembre <https://bit.ly/3zTnXOK> Moreau G. (2008), Apprentissage. Une singulière métamorphose, Formation emploi, n.101, pp.119-133

OFS - Office fédéral de la statistique (2018), Transitions après un titre du degré secondaire II et intégration sur le marché du travail, Neuchâtel, Office Fédéral de la Statistique

O’Reilly J., Leschke J., Ortlieb R., Seeleib-Kaiser M., Villa P. (2018), Comparing youth transitions in Europe. Joblessness, insecurity, institutions, and inequality, in O'Reilly J., Leschke J., Ortlieb R., Seeleib-Kaiser M., Villa P. (eds.), Youth Labor in Transitions. Inequalities, Mobility and Policies in Europe, Oxford, Oxford University Press, pp.1-29

Piopiunik M., Ryan P. (2012), Improving the Transition Between Education/Training and the Labour Market. What Can We Learn From Various National Approaches?, EENEE Analytical Report n.13, Munich, EENEE

Quintini G., Martin J.P., Martin S. (2007), The Changing Nature of the School-to-Work Transition Process in OECD Countries, IZA Discussion Paper n.2582, Bonn, IZA <https://bit.ly/3ieBjPi>

Ryan P. (2001), The School-to-Work Transition. A Cross-National Perspective, Journal of Economic Literature, 39, n.1, pp.34-92 Salerno G.M. (2020), La disomogeneità dell'offerta formativa. Riflessioni e proposte, Bene comune. La Rivista, n.1-2, pp.32-36

Scarpetta S., Sonnet A., Manfredi T. (2010), Rising Youth Unemployment During The Crisis. How to Prevent Negative Long-term Consequences on a Generation?, OECD Social, Employment and Migration Working Papers n.106, Paris, OECD Publishing

Solga H., Protsch P., Ebner C., Brzinsky-Fay C. (2014), The German vocational education and training system. Its institutional configuration, strengths, and challenges, WZB Discussion Paper n.502, Berlin, Wissenschaftszentrum Berlin für Sozialforschung

Thelen K. (2007), Contemporary challenges to the German vocational training system, Regulation \& Governance, n.1, pp.247-260

Tiraboschi M. (2016), L'apprendistato dopo il Jobs Act, in Carinci F. (a cura di), Jobs Act: un primo bilancio. Atti dell’XI Seminario di Bertinoro-Bologna del 22-23 ottobre 2015, ADAPT Labour Studies e-Book series n.54, Modena, ADAPT University Press, pp.306-330<https://bit.ly/2ZE4EMx>

Verick S. (2009), Who is Hit Hardest during a Financial Crisis? The Vulnerability of Young Men and Women to Unemployment in an Economic Downturn, IZA Discussion Paper n.4359, Bonn, IZA

Zagardo G. (2019), La leFP nelle Regioni. Tra consolidamento e stasi, Quaderni CNOS-FAP n.9, Roma, CNOS-FAP <https://bit.ly/3DOvrBi>

\begin{abstract}
Sandra D’Agostino
s.dagostino@inapp.org

Ricercatrice Inapp. Per oltre vent'anni ha svolto attività di ricerca in materia di politiche della formazione, prestando la propria opera anche a supporto dei decisori a livello nazionale e territoriale. Fra i vari ambiti di studio affrontati, I'analisi degli strumenti dell'alternanza è quello al quale si è dedicata più a lungo. In Inapp ha ricoperto il ruolo di responsabile di unità operativa e responsabile di Struttura. Fra le pubblicazioni recenti: Nuove tutele per i lavoratori: il diritto soggettivo alla formazione. Francia e Italia a confronto, Professionalità Studi, III, n.2, 2020; La via italiana al duale: opportunità e criticità, Economia e Società Regionale, n.1, 2020.
\end{abstract}

\title{
Silvia Vaccaro
}

s.vaccaro@inapp.org

Ricercatrice Inapp. Svolge da oltre venti anni indagini sulle politiche formative e attività di consulenza scientifica. Ha svolto in particolare ricerche sugli strumenti di alternanza scuola-lavoro, con specifico riferimento all'apprendistato, sulla formazione continua e sulle politiche di apprendimento permanente in Italia e in Europa. Fra le pubblicazioni recenti: Nuove tutele per i lavoratori: il diritto soggettivo alla formazione. Francia e Italia a confronto, Professionalità Studi, III, n.2, 2020; La via italiana al duale: opportunità e criticità, Economia e Società Regionale, n.1, 2020. 